\title{
PERFORMANCES OF UNKNOWN INPUT OBSERVERS FOR CHAOTIC LPV MAPS IN A STOCHASTIC CONTEXT
}

\author{
G. Millérioux and J. Daafouz. \\ Centre de Recherche en Automatique de Nancy (CRAN), \\ Université Henri Poincaré Nancy 1, Institut National de Polytechnique de Lorraine \\ e-mail: millerioux@esstin.uhp-nancy.fr, jdaafouz@ensem.inpl-nancy.fr \\ WWW: http://www.cran.uhp-nancy.fr
}

\begin{abstract}
Jointly modeling chaotic maps as LPV systems and using Unknown Input Observers for retrieving the information in a secure communication scheme has previously been motivated in a deterministic context [1]. In this paper, some new theoretical results from a control theory point of view, concerning the design in a stochastic and so more realistic context of Unknown Input Observers for chaotic LPV systems is provided. The design of such observers is expressed in terms of the resolution of a finite set of Matrix Inequalities constraints and guarantees some prescribed performances on the state reconstruction error.
\end{abstract}

\section{INTRODUCTION}

The well-known practical interest of chaos synchronization lies in the potential applications in communications and more specifically in the possibilities of encoding or masking messages by embedding the information into the dynamics of a chaotic system. The information to be masked plays the role of an external input for the dynamical system and is not transmitted to the receiver. Hence, the receiver system must be designed such that the information can be unmasked, given the only available output data consisting of a function of the state vector. In [1], a brief survey of the main approaches proposed in the literature is given. Then, a novel approach based on Unknown Input Observer (UIO) for a noise-free context is proposed.

In a deterministic context, Unknown Input Observers have been largely investigated for linear systems [2][3][4][5]. On the other hand, in a stochastic context, there does not exist a lot of results. For timeinvariant systems, we can mention the works of [6] while for linear time-varying systems, the reader can refer to [7], but none of those classes of systems can exhibit chaotic behaviors and so have no interest for chaos-based communications purposes.

The aim of this paper is to state some new results concerning the design of Unknown Input Observers for Linear Parameter Varying Systems in a stochastic context. The interest of LPV systems lies in the fact that a large amount of chaotic systems enter this class. Furthermore, the UIO design guaranteeing some prescribed performances can be carried out in a tractable way by solving Matrix Inequalities.

Notation : $(\bullet)^{T}$ stands for the symmetric block of a positive definite matrix, $\mathbf{0}$ and $\mathbf{1}$ stand for the zero and the identity matrix of appropriate size. $\left\|x_{k}\right\|=\sqrt{x_{k}^{T} x_{k}},\|x\|_{\infty}=\sup _{k \geq 0}\left\|x_{k}\right\|$ and $\|x\|_{2}=$ $\sqrt{\sum_{k=0}^{\infty} x_{k}^{T} x_{k}}$.

\section{UNKNOWN INPUT OBSERVERS FOR LPV SYSTEMS}

Consider the general state space realization of LPV discrete-time systems in a noisy context.

$$
\left\{\begin{array}{l}
x_{k+1}=A\left(\rho_{k}\right) x_{k}+B u_{k}+E v_{k} \\
y_{k}=C x_{k}+D v_{k}
\end{array}\right.
$$

where $x_{k} \in \mathbb{R}^{n}, y_{k} \in \mathbb{R}^{m}, A \in \mathbb{R}^{n \times n}, B \in \mathbb{R}^{n \times r}$, $C \in \mathbb{R}^{m \times n}, D \in \mathbb{R}^{m \times r} . u_{k} \in \mathbb{R}^{r}$ is the input, $v_{k} \in$ $\mathbb{R}^{l}$ is the disturbance acting on the dynamics through $E$ and acting on the measurement through $D . A$ is of class $C^{1}$ with respect to the entries of a $L$-dimensional time-varying parameter vector $\rho_{k}=\left(\rho_{k}^{1}, \ldots, \rho_{k}^{L}\right)^{T}$. In [8], it has been shown that a lot of chaotic maps can be modeled by LPV discrete-time systems with $\rho_{k}$ being a function of the state vector $x_{k}$. Since $x_{k}$ evolves chaotically, $\rho_{k}$ is bounded in a hypercube $\Theta$. As a result, $A$ lies in a compact set which can always be embedded in a polytope, that is :

$$
A\left(\rho_{k}\right)=\sum_{i=1}^{N} \xi_{k}^{i}\left(\rho_{k}\right) A_{i}
$$

where the $A_{i}$ 's correspond to the vertices of the convex hull $\mathbf{C o}\left\{A_{1}, \ldots, A_{N}\right\}$. The $\xi_{k}$ 's belong to the compact set $\mathcal{S}=\left\{\mu_{k} \in \mathbb{R}^{N}, \mu_{k}=\right.$ $\left(\mu_{k}^{1}, \ldots, \mu_{k}^{N}\right)^{T}, \mu_{k}^{i} \geq 0 \forall i$ and $\left.\sum_{i=1}^{N} \mu_{k}^{i}=1\right\}$ and 
they can always be expressed as functions of class $C^{1}$ with respect to the $\rho_{k}$ 's. The advantage of such a decomposition lies in the fact that the design problems turn into the resolution of a finite set of constraints involving only the vertices of the convex hull.

For secure communication purposes, $u_{k}$ plays the role of the information to be masked and acts as an unknown input. $y_{k}$ is the signal transmitted to the receiver. The structure of the required Unknown Input Observers for the recovering of $u_{k}$ is reminded from [1].

$\hat{x}_{k+1}=\left(P A\left(\rho_{k}\right)-L\left(\rho_{k}\right) C\right) \hat{x}_{k}+L\left(\rho_{k}\right) y_{k}+Q y_{k+1}$

with $P=\mathbf{1}_{n}-Q C$ and $L\left(\rho_{k}\right)=\sum_{i=1}^{N} \xi_{k}^{i}\left(\rho_{k}\right) L_{i}$. The gains $Q$ and $L_{i}$ 's $(i=1, \ldots, N)$ are unknown matrices to be computed.

From (1) and (3), it is straightforward to show that the state reconstruction error $\epsilon_{k} \triangleq x_{k}-\hat{x}_{k}$ is governed by :

$$
\epsilon_{k+1}=\mathcal{A}\left(\rho_{k}\right) \epsilon_{k}+P B u_{k}+\mathcal{B}\left(\rho_{k}\right) v_{k}-Q D v_{k+1}
$$

with $\mathcal{A}\left(\rho_{k}\right)=\sum_{i=1}^{N} \xi_{k}^{i}\left(P A_{i}-L_{i} C\right)$ and $\mathcal{B}\left(\rho_{k}\right)=$ $\sum_{i=1}^{N} \xi_{k}^{i}\left(P E-L_{i} D\right)$.

Before dealing with the performances on the state reconstruction when disturbances act on the system, it is necessary to remind how the global stability of the null solution of (4) can be guaranteed when $v_{k}=0$. Some details can be found in [1].

Theorem 1. The global stability of the null solution of (4) with $v_{k}=0$ is ensured if

i) $\operatorname{rank}(C B)=\operatorname{rank}(B)=r$,

ii) there exist symmetric matrices $P_{i}$, matrices $F_{i}$ and $G_{i}$ such that, $\forall(i, j) \in\{1, \ldots, N\} \times\{1, \ldots, N\}$, the following set of Linear Matrix Inequalities is feasible.

$$
\left[\begin{array}{cc}
P_{i} & (\bullet)^{T} \\
G_{i} P A_{i}-F_{i} C & G_{i}^{T}+G_{i}-P_{j}
\end{array}\right]>0
$$

The time-varying gain is given by $L\left(\rho_{k}\right)=$ $\sum_{i=1}^{N} \xi_{k}^{i} L_{i}$ with $L_{i}=G_{i}^{-1} F_{i}$.

Proof: On one hand, according to the definition of $P$, the equality $P B=0$ entails that $Q$ must be subject to

$$
B=Q C B
$$

and $i$ ) ensures the existence of the solution $Q$ of (6). Its general expression is :

$$
Q=B(C B)^{\dagger}+Y\left(\mathbf{1}_{m}-(C B)(C B)^{\dagger}\right)
$$

with $Y$ an arbitrary matrix. Then, whenever $Q$ satisfies (7), $P B=0$ and so (4) turns into an input independent dynamics :

$$
\epsilon_{k+1}=\mathcal{A}\left(\rho_{k}\right) \epsilon_{k}+\mathcal{B}\left(\rho_{k}\right) v_{k}-Q D v_{k+1}
$$

On the other hand, the proof follows a reasoning similar to the one carried out in [9]. All the relations are valid $\forall(i, j) \in\{1, \ldots, N\} \times\{1, \ldots, N\}$.

a) Since $P_{j}$ is strictly positive, one has :

$$
G_{i} P_{j}^{-1} G_{i}^{T} \geq G_{i}+G_{i}^{T}-P_{j}
$$

b) Substitute $F_{i}$ by $G_{i} L_{i}$ in (5) and take into account the inequality above yields :

$$
\left[\begin{array}{cc}
P_{i} & (\bullet)^{T} \\
G_{i}\left(P A_{i}-L_{i} C\right) & G_{i} P_{j}^{-1} G_{i}^{T}
\end{array}\right]>0
$$

which is equivalent to

$$
\mathbb{H} \cdot\left[\begin{array}{cc}
P_{i} & (\bullet)^{T} \\
P_{j}\left(P A_{i}-L_{i} C\right) & P_{j}
\end{array}\right] \cdot \mathbb{H}^{T}
$$

with

$$
\mathbb{H}=\left[\begin{array}{cc}
\mathbf{1} & \mathbf{0} \\
\mathbf{0} & G_{i} P_{j}^{-1}
\end{array}\right]
$$

and so to

$$
\left[\begin{array}{cc}
P_{i} & (\bullet)^{T} \\
P_{j}\left(P A_{i}-L_{i} C\right) & P_{j}
\end{array}\right]>0
$$

since $G_{i}$ and $P_{j}$ are full rank matrices.

c) For each $i=1, \ldots, N$, multiply the corresponding $j=1, \ldots, N$ inequalities (11) by $\xi_{k+1}^{j}$ and sum. Then, multiply the resulting $i=1, \ldots, N$ inequalities by $\xi_{k}^{i}$ and sum again. We obtain :

$$
\left[\begin{array}{cc}
\mathcal{P}_{k} & (\bullet)^{T} \\
\mathcal{P}_{k+1} \mathcal{A} & \mathcal{P}_{k+1}
\end{array}\right]>0
$$

with $\mathcal{P}_{k}=\sum_{i=1}^{N} \xi_{k}^{i} P_{i}$ and $\mathcal{P}_{k+1}=\sum_{i=1}^{N} \xi_{k+1}^{i} P_{i}$. Applying the Schur complement formula gives :

$$
\mathcal{A}^{T} \mathcal{P}_{k+1} \mathcal{A}-\mathcal{P}_{k}<0 \quad \forall k
$$

It is shown in [9] that $V: \mathbb{R}^{n} \rightarrow \mathbb{R}^{+}$, a function defined by $V\left(z_{k}, \xi_{k}\right)=z_{k}^{T} \mathcal{P}_{k} z_{k}$ with $\mathcal{P}_{k}=\sum_{i=1}^{N} \xi_{k}^{i} P_{i}$ and $\xi_{k} \in \mathcal{S}$ acts as a Lyapunov function for (4) when $v_{k}=0$ and ensures the poly-quadratic stability of (4) which is sufficient to global asymptotical stability. This completes the proof.

In the forthcoming sections, the case $v_{k} \neq 0$, that is the stochastic context, is considered and constitutes the main result of the paper. 


\section{OBSERVER DESIGN WITH PRESCRIBED PERFORMANCES}

\section{A. Bounded $\mathcal{L}_{2}$ gain}

We define the upper bound denoted $\sigma$ of the $\mathcal{L}_{2}$ gain as a scalar verifying :

$$
\sup _{\|\bar{v}\|_{2} \neq 0} \frac{\|z\|_{2}}{\|\bar{v}\|_{2}}<\sigma
$$

where $z_{k}=\tilde{C} \epsilon_{k}, \bar{v}_{k}=\left[\begin{array}{ll}v_{k}^{T} & v_{k+1}^{T}\end{array}\right]^{T}$.

Theorem 2. The $\mathcal{L}_{2}$ gain corresponding to (4) with $v_{k} \neq 0$ is less than $\sigma$ if

i) $\operatorname{rank}(C B)=\operatorname{rank}(B)=r$,

ii) there exist symmetric matrices $P_{i}$, matrices $F_{i}$ and $G_{i}$ such that $\forall(i, j) \in\{1, \ldots, N\} \times\{1, \ldots, N\}$, the set (21) of Linear Matrix Inequalities is feasible.

The time-varying gain is given by $L\left(\rho_{k}\right)=$ $\sum_{i=1}^{N} \xi_{k}^{i} L_{i}$ with $L_{i}=G_{i}^{-1} F_{i}$.

Proof: For the same reason motivated in the proof of Theorem 1, condition $i$ ) ensures the existence of a matrix $Q$ such that $P B=0$ holds and turns (4) into an input independent dynamics. Besides, define the matrices

$$
\mathbb{G}_{i}=\left[\begin{array}{ccc}
G_{i} & \mathbf{0} & \mathbf{0} \\
\mathbf{0} & \sigma \mathbf{1} & \mathbf{0} \\
\mathbf{0} & \mathbf{0} & \sigma \mathbf{1}
\end{array}\right], \quad \mathbb{P}_{i}=\left[\begin{array}{ccc}
P_{i} & \mathbf{0} & \mathbf{0} \\
\mathbf{0} & \sigma \mathbf{1} & \mathbf{0} \\
\mathbf{0} & \mathbf{0} & \sigma \mathbf{1}
\end{array}\right]
$$

and

$$
\mathbb{M}_{i}=\left[\begin{array}{ccc}
P A_{i}-L_{i} C_{i} & P E-L_{i} D & -Q D \\
\sigma^{-1} \tilde{C} & \mathbf{0} & \mathbf{0} \\
\mathbf{0} & \mathbf{0} & \mathbf{0}
\end{array}\right]
$$

(21) can be rewritten :

$$
\left[\begin{array}{cc}
\mathbb{P}_{i} & (\bullet)^{T} \\
\mathbb{G}_{i} \mathbb{M}_{i}^{T} & \mathbb{G}_{i}+\mathbb{G}_{i}^{T}-\mathbb{P}_{j}
\end{array}\right]>\mathbf{0}
$$

Following the same three steps $a$ ) to $c$ ) as in the proof of Theorem 1, feasibility of (15) implies that

$$
\mathbb{M}^{T} \mathbb{P}_{k+1} \mathbb{M}-\mathbb{P}_{k}<0
$$

with

$\mathbb{P}_{k}=\left[\begin{array}{ccc}\mathcal{P}_{k} & \mathbf{0} & \mathbf{0} \\ \mathbf{0} & \sigma \mathbf{1} & \mathbf{0} \\ \mathbf{0} & \mathbf{0} & \sigma \mathbf{1}\end{array}\right], \mathbb{P}_{k+1}=\left[\begin{array}{ccc}\mathcal{P}_{k+1} & \mathbf{0} & \mathbf{0} \\ \mathbf{0} & \sigma \mathbf{1} & \mathbf{0} \\ \mathbf{0} & \mathbf{0} & \sigma \mathbf{1}\end{array}\right]$

and

$$
\mathbb{M}=\left[\begin{array}{ccc}
\mathcal{A} & \mathcal{B} & -Q D \\
\sigma^{-1} \mathbf{1} & \mathbf{0} & \mathbf{0} \\
\mathbf{0} & \mathbf{0} & \mathbf{0}
\end{array}\right]
$$

Equation (16) can be rewritten like (22). Then, multiply left and right respectively by $\left[\begin{array}{ll}\epsilon_{k} & \bar{v}_{k}\end{array}\right]$ and its transpose gives :

$V\left(\epsilon_{k+1}, \xi_{k+1}\right)-V\left(\epsilon_{k}, \xi_{k}\right)+\sigma^{-1}\left(\tilde{C} \epsilon_{k}\right)^{T}\left(\tilde{C} \epsilon_{k}\right)-\sigma \bar{v}_{k}^{T} \bar{v}_{k}<0$

Consider (17) from $k=0$ to $N$ and sum leads to:

$V\left(\epsilon_{N+1}, \xi_{N+1}\right)+\sigma^{-1} \sum_{k=0}^{N}\left(\tilde{C} \epsilon_{k}\right)^{T}\left(\tilde{C} \epsilon_{k}\right)-\sigma \sum_{k=0}^{N} \bar{v}_{k}^{T} \bar{v}_{k}<0$

Yet, $V\left(\epsilon_{N+1}, \xi_{N+1}\right)=\epsilon_{N+1}^{T} \mathcal{P}_{k} \epsilon_{N+1}>0$. Hence :

$$
\sigma^{-1} \sum_{k=0}^{N}\left(\tilde{C} \epsilon_{k}\right)^{T}\left(\tilde{C} \epsilon_{k}\right)<\sigma \sum_{k=0}^{N} \bar{v}_{k}^{T} \bar{v}_{k}
$$

When $N$ tends toward infinity, this relation is equivalent to (14). This completes the proof.

\section{B. Peak-to-peak gain}

Let $\nu$ be the upper bound of the peak-to-peak gain defined as the ratio between $\|\epsilon\|_{\infty}$ and $\|\bar{v}\|_{\infty}$

$$
\sup _{0<\|\bar{v}\|_{\infty}<\infty} \frac{\|\epsilon\|_{\infty}}{\|\bar{v}\|_{\infty}}<\nu
$$

$$
\left[\begin{array}{cccccc}
P_{i} & (\bullet)^{T} & (\bullet)^{T} & (\bullet)^{T} & (\bullet)^{T} & (\bullet)^{T} \\
\mathbf{0} & \sigma \mathbf{1} & (\bullet)^{T} & (\bullet)^{T} & (\bullet)^{T} & (\bullet)^{T} \\
\mathbf{0} & \mathbf{0} & \sigma \mathbf{1} & (\bullet)^{T} & (\bullet)^{T} & (\bullet)^{T} \\
G_{i} P A_{i}-F_{i} C & G_{i} P E-F_{i} D & -G_{i} Q D & G_{i}+G_{i}^{T}-P_{j} & (\bullet)^{T} & (\bullet)^{T} \\
\tilde{C} & \mathbf{0} & \mathbf{0} & \mathbf{0} & \sigma \mathbf{1} & (\bullet)^{T} \\
\mathbf{0} & \mathbf{0} & \mathbf{0} & \mathbf{0} & \mathbf{0} & \sigma \mathbf{1}
\end{array}\right]>0
$$

$$
\left[\begin{array}{ccc}
\mathcal{A}^{T} \mathcal{P}_{k+1} \mathcal{A}-\mathcal{P}_{k}+\sigma^{-1} \tilde{\mathcal{C}}^{T} \tilde{\mathcal{C}} & (\bullet)^{T} & (\bullet)^{T} \\
\mathcal{B}^{T} \mathcal{P}_{k+1} \mathcal{A} & \mathcal{B}^{T} \mathcal{P}_{k+1} \mathcal{B}-\sigma \mathbf{1} & (\bullet)^{T} \\
-(Q D)^{T} \mathcal{P}_{k+1} \mathcal{A} & -(Q D)^{T} \mathcal{P}_{k+1} \mathcal{B} & (Q D)^{T} \mathcal{P}_{k+1}(Q D)-\sigma \mathbf{1}
\end{array}\right]>0
$$


Theorem 3. The peak-to-peak gain corresponding to (4) with $v_{k} \neq 0$ is less than $\nu>0$ if

i) $\operatorname{rank}(C B)=\operatorname{rank}(B)=r$,

ii) there exist symmetric positive definite matrices $P_{i}$, matrices $G_{i}$, scalars $\left.\lambda \in\right] 0,1[, \mu>0$ such that, $\forall(i, j) \in\{1, \ldots, N\} \times\{1, \ldots, N\}$, the Matrix Inequalities (29) are fulfilled.

The time-varying gain is given by $L\left(\rho_{k}\right)=$ $\sum_{i=1}^{N} \xi_{k}^{i} L_{i}$ with $L_{i}=G_{i}^{-1} F_{i}$.

Proof: For the same reason motivated in the proof of Theorem 1, condition $i$ ) turns (4) into an input independent dynamics. On one hand, again, considering the first inequality of (29) and following the same steps from $a$ ) to $c$ ) as in the proof of Theorem 1 yields :

$$
\left[\begin{array}{cccc}
(1-\lambda) \cdot \mathcal{P}_{k} & (\bullet)^{T} & (\bullet)^{T} & (\bullet)^{T} \\
\mathbf{0} & \mu \mathbf{1} & (\bullet)^{T} & (\bullet)^{T} \\
\mathbf{0} & \mathbf{0} & \mu \mathbf{1} & (\bullet)^{T} \\
\mathcal{P}_{k+1} \mathcal{A} & \mathcal{P}_{k+1} \mathcal{B} & -\mathcal{P}_{k+1} Q D & \mathcal{P}_{k+1}
\end{array}\right]>0
$$

Applying the Schur complement formula and somes basic manipulations yields (30). Then, multiply (30) left and right respectively by $\left[\epsilon_{k} \quad \bar{v}_{k}\right]$ and its transpose, entails that :

$$
V\left(\epsilon_{k+1}, \xi_{k+1}\right)<(1-\lambda) V\left(\epsilon_{k}, \xi_{k}\right)+\mu\left\|\bar{v}_{k}\right\|^{2} \quad \forall k
$$

Applying the Gronwall-lemma in the discrete case gives :

$$
V\left(\epsilon_{k}, \xi_{k}\right)<\frac{\mu}{\lambda}\|\bar{v}\|_{\infty}^{2} \quad \forall k
$$

On the other hand, multiplying the second inequality of (29) by $\xi_{k}^{i}$ and sum from $i=1$ to $N$ gives :

$$
\left[\begin{array}{cc}
\lambda \mathcal{P}_{k}-\frac{1}{\nu} \mathbf{1} & \mathbf{0} \\
\mathbf{0} & (\nu-\mu) \mathbf{1}
\end{array}\right]>0
$$

Besides, multiply (26) left and right respectively by $\left[\begin{array}{ll}\epsilon_{k} & \bar{v}_{k}\end{array}\right]$ and its transpose leads to :

$$
\frac{1}{\nu}\left\|\epsilon_{k}\right\|^{2}<\lambda V\left(\epsilon_{k}, \xi_{k}\right)+(\nu-\mu)\left\|v_{k}\right\|^{2} \quad \forall k
$$

Finally, combining (25) and (27) and taking into account that $\nu-\mu>0$ from the second inequality of (29) leads to :

$$
\|\epsilon\|_{\infty}^{2}<\nu^{2}\|v\|_{\infty}^{2}
$$

And yet, (28) is equivalent to (20).

\section{Remark}

Note that the Matrix Inequalities coresponding to the peak-to-peak performances are not linear unlike the ones related to the $\mathcal{L}_{2}$ gain. They involve a product of two unknowns, say $\lambda$ and $P_{i}$, and a nonlinear dependence on $\nu$ which prevents the convexity.

\section{REFERENCES}

[1] G. Millerioux and J. Daafouz. Unknown input observers for message-embedded chaos synchronization of discrete-time systems. International Journal of Bifurcation and Chaos, 14(4), April 2004.

[2] M. Darouach, M. Zazadinski, and S. J. Xu. Full-order observers for linear systems with unknown inputs. IEEE Trans. on Automatic Control, 39(3):606-609, March 1994.

[3] S-K. Chang, W-T. You, and P-L. Hsu. Design of general structured observers for linear systems with unknown inputs. J. Franklin Inst., 334(2):213-232, 1997.

[4] F. Yang and R. W. Wilde. Observers for linear systems with unknown inputs. IEEE Trans. on Automatic Control, 33(7):677-681, July 1988.

[5] Y. Guan and M. Saif. A novel approach to the design of unknown input observers. IEEE Trans. on Automatic Control, 36(5):632-635, May 1991.

[6] Nikoukhah R. Innovations generation in the presence of unknown inputs: application to robust failure detection. Automatica, 30:1851-1867, 1994.

[7] M. Darouach, M. Zazadinski, and M. Boutayeb. Extension to minimum variance estimation for systems with unknown inputs. Automatica, 39:867-876, 2003.

[8] G. Millerioux and J. Daafouz. Polytopic observer for global synchronization of systems with output measurable nonlinearities. International Journal of Bifurcation and Chaos, 13(3):703-712, March 2003.

[9] J. Daafouz and J. Bernussou. Parameter dependent lyapunov functions for discrete time systems with time varying parametric uncertainties. Systems and Control Letters, 43:355359,2001

$$
\left[\begin{array}{cccc}
(1-\lambda) \cdot P_{i} & (\bullet)^{T} & (\bullet)^{T} & (\bullet)^{T} \\
\mathbf{0} & \mu \mathbf{1} & (\bullet)^{T} & (\bullet)^{T} \\
\mathbf{0} & \mathbf{0} & \mu \mathbf{1} & (\bullet)^{T} \\
G_{i} P A_{i}-F_{i} C & G_{i} P E-F_{i} D & -G_{i} Q D & G_{i}+G_{i}^{T}-P_{j}
\end{array}\right]>0, \quad\left[\begin{array}{cc}
\lambda P_{i}-\frac{1}{\nu} \mathbf{1} & (\bullet)^{T} \\
\mathbf{0} & (\nu-\mu) \mathbf{1}
\end{array}\right]>0
$$

$$
\left[\begin{array}{ccc}
\mathcal{A}^{T} \mathcal{P}_{k+1} \mathcal{A}-(1-\lambda) \mathcal{P}_{k} & (\bullet)^{T} & (\bullet)^{T} \\
\mathcal{B}^{T} \mathcal{P}_{k+1} \mathcal{A} & -\mu \mathbf{1}+\mathcal{B}^{T} \mathcal{P}_{k+1} \mathcal{B} & (\bullet)^{T} \\
-(Q D)^{T} \mathcal{P}_{k+1} \mathcal{A} & -(Q D)^{T} \mathcal{P}_{k+1} \mathcal{B} & -\mu \mathbf{1}+(Q D)^{T} \mathcal{P}_{k+1} Q D
\end{array}\right]<0
$$

